\title{
Individual applicator for brachytherapy for various sites of superficial malignant lesions
}

\author{
Łukasz Kowalik, MSc, Jarosław Łyczek, PhD, MD, Marcin Sawicki, MSc, Damian Kazalski, MD \\ Brachytherapy Department, Hospital in Brzozów, Podkarpacki Oncology Centre, Brzozów, Poland
}

\begin{abstract}
The aim of this paper is to discuss brachytherapy treatment and individual applicators suitable for unfavorably localized superficial malignant lesions. Techniques for manufacturing an individual applicator and clinical examples of its use for various locations of cancer are presented. This techniques are based on individual size and shape of the tumour. CT-planning make the technique adequate for individual patient history and type of tumour. Featured techniques seems to be very useful and easy to performed.

Key words: HDR brachytherapy, individual applicators, superficial lesions.

\section{Purpose}

Several types of brachytherapy applicators are suitable for superficial malignant skin lesions depending on their size and depth. Small skin changes (up to $3 \mathrm{~cm}$ ) and superficial cancer (to $0.5 \mathrm{~cm}$ ) are usually treated via Leipzig ${ }^{\circledR}$ or Valencia ${ }^{\circledR}$ skin applicators [1]. Freiburg Flap ${ }^{\circledR}$ applicator or $\mathrm{HAM}^{\circledR}$ (all applicators made by Nucletron, an Elekta company, Elekta AB, Stockholm, Sweden) are commonly used for extensive skin lesions on uncovered body parts with infiltration depth less than $1 \mathrm{~cm} \mathrm{[2].} \mathrm{However,} \mathrm{skin} \mathrm{as} \mathrm{well}$ as mucosal cancers located in unfavorably localized areas and lesions with irregularly shaped surface, require special applicators prepared individually for each patient [3]. Described cases were uniquely located and because of that, it was difficult to prepare treatment plans for external beam radiation therapy (EBRT). Also age and general condition of the patients played impediment role in realization of the treatment plan. From that reasons, local brachytherapy seemed to be most repeatability.

\section{Technique of manufacturing an individual applicator}

The target volume with adequate margins was defined by a radiation oncologist based on physical examinations and histopathological assessment of tissue. Also, brachytherapy specialists paid special attention to organs at risk (OARs), which should be protected from ionizing radiation. The surface mould was made by a physicist, who assessed its range, size, and fixation methods if possible for a given location e.g.: nose, ear, and fingers. Before we decided the shape of the applicator and manufacturing techniques, it was necessary to select an organ or anatomical site stable and unchangeable during the whole process of treatment (nose, lip, outer ear). Patient's skin was cleaned and dried. Then alginate impression material (KROMOPAN $100^{\circledR}$, LASCOD S.p.A. Florence, Italy) was prepared according to the manufacturer's instructions and placed on patient's skin. It should be taken into account that the material changes its colour during the process of polymerisation. In order to obtain the best possible impression of patient's shape, the paste consistency should be adjusted individually.

Setting time was approximately 2 minutes. Then it's thickness was assessed whether it was sufficient, i.e. minimum thickness between 1 and $2 \mathrm{~cm}$. If possible, another layer of alginate material was added using the same technique. Next, the impression was removed from patient's skin and the specialist evaluated the accuracy of patient's shape reconstruction [4].

The impression was later used for a gypsum cast of the patient. The cast should be positioned properly in order to reflect all natural curves of the body. Gypsum material was also prepared according to the manufacturer's instructions. The first layer should not be too thick, otherwise small cavities in the impression might not be filled with gypsum. Then another layer was poured over the cast. This step should be performed carefully, because the cast must be placed in a special holder. As the cast hardens into a rigid form, the alginate impression can be removed gently. Corrections of small deficiencies of the cast were allowed. Usually gypsum solidifies completely within 24 hours. This period may be shortened if the cast is stored in an air-conditioned room. The final step is the preparation of a surface applicator from skin safe silicone mass.

The process of manufacturing the applicator included the following steps: (a) lead plates were placed on the gypsum cast in order to protect organs at risk (eyeball, mucosa, etc). 

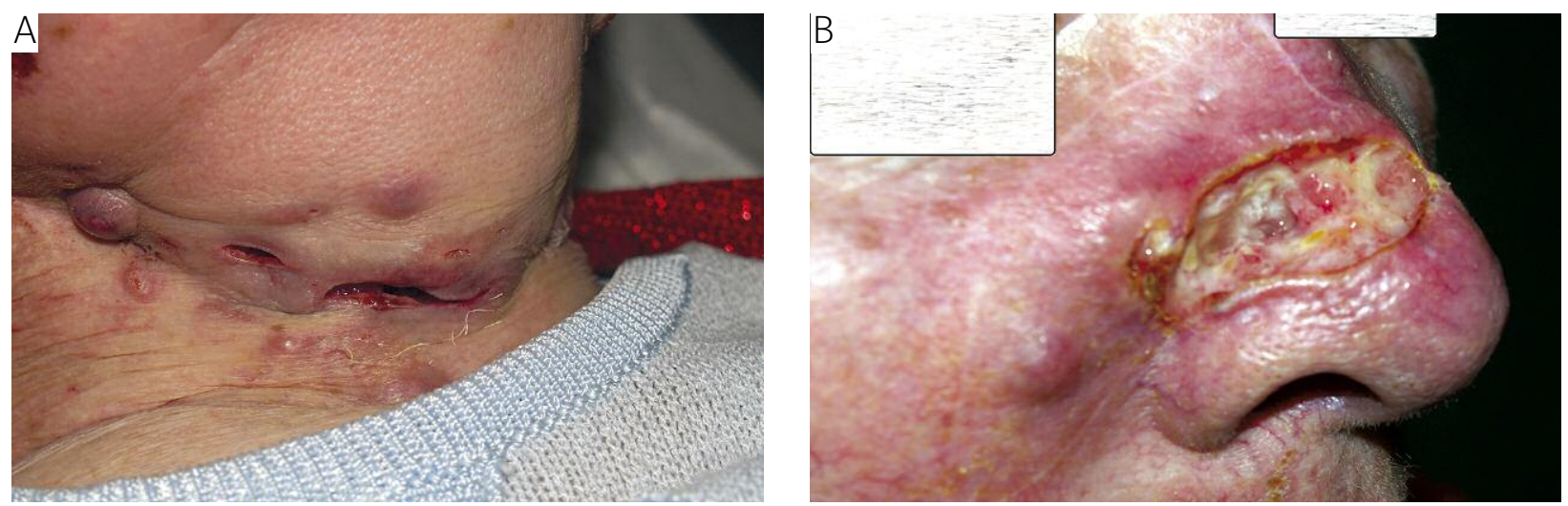

Fig. 1A-B. Patient with multifocal head and neck cancer (case 1)

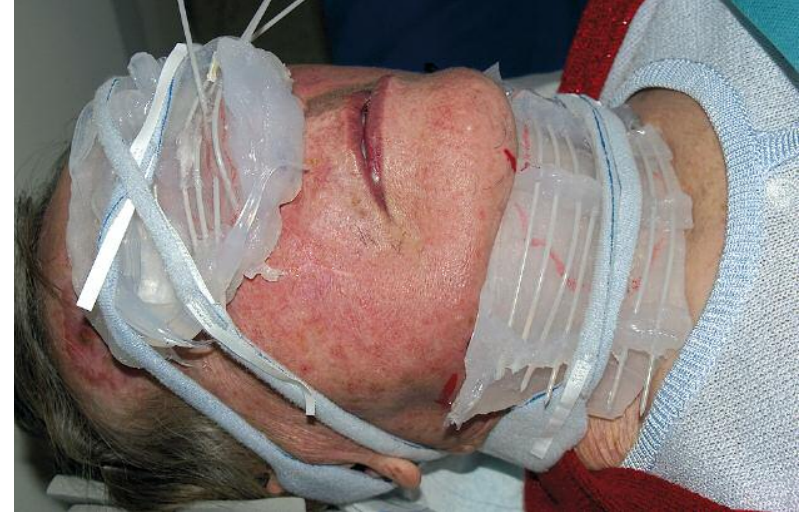

Fig. 2. Silicone impressions for a patient with multifocal head and neck cancer (case 1)
The thickness of these plates should be selected in accordance to the radioactive source used for brachytherapy, i.e. $6 \mathrm{~mm}$ for ${ }^{192} \mathrm{Ir}$ and $10 \mathrm{~mm}$ for ${ }^{60} \mathrm{Co}$ [5]. Lead plates must be removable. They should not be present during CT scans if we want to limit the number of artifacts in CT images. The plates must be replaced before the first fraction of brachytherapy; (b) a layer of silicone was placed on the gypsum cast - it's thickness directly above the planning target volume (PTV) was approximately $5 \mathrm{~mm}$. In the remaining area it was more than $5 \mathrm{~mm}$ and more than $10 \mathrm{~mm}$ in the area where the applicator would be fixed/stabilised; (c) when the surface of the applicator was dry, OncoSmart ProGuide Needles ${ }^{\circledR}$ (Nucletron, an Elekta company, Elekta AB, Stockholm, Sweden) were inserted above the PTV. The needles were parallel to each other and the distance between adjacent nee-

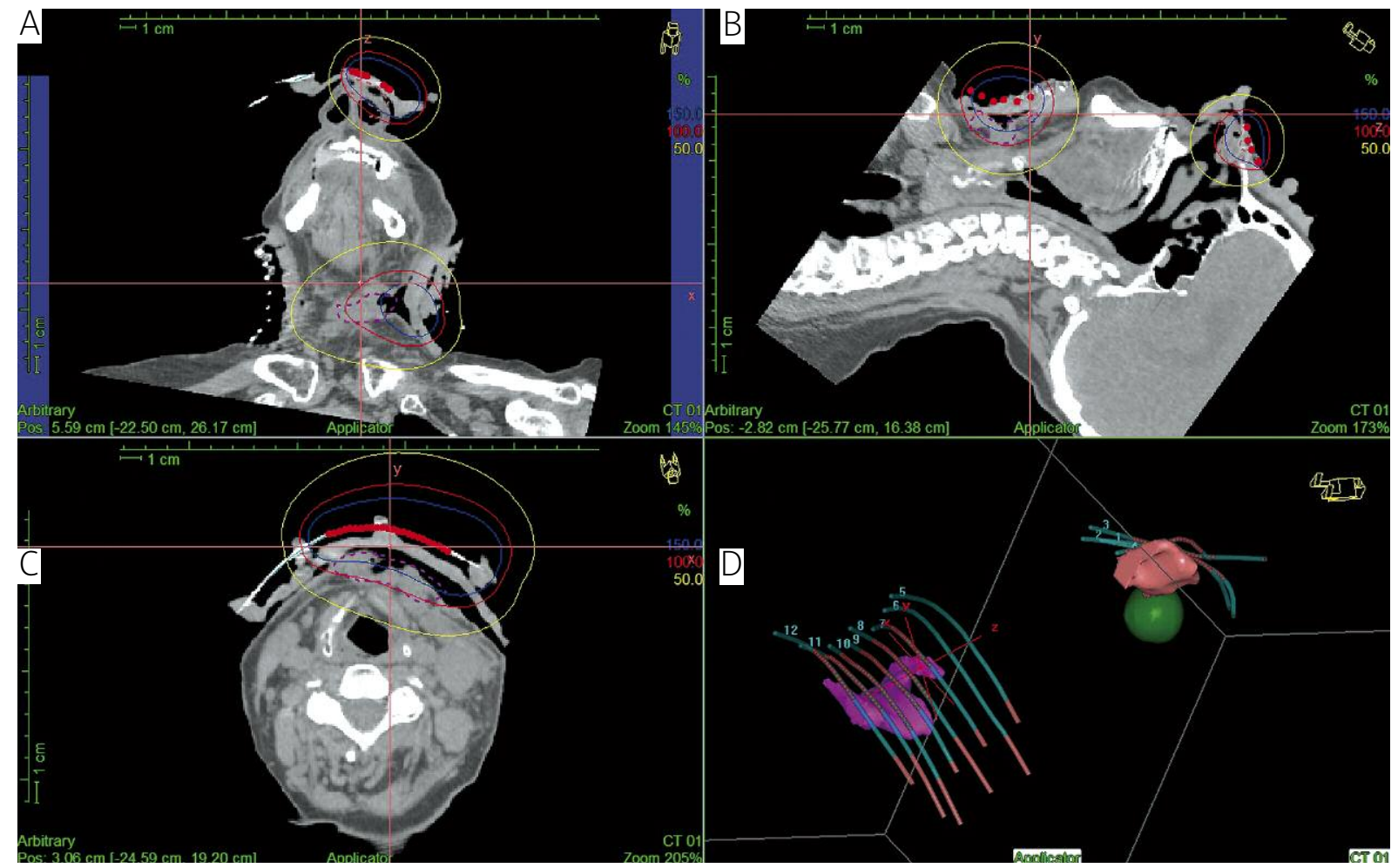

Fig. 3. A patient with multifocal head and neck cancer - CT images and reconstructions in different planes (A, B, C) and a 3D reconstruction (D) (case 1) 
dles ranged from 3 to $7 \mathrm{~mm}$. The needles covered the whole target volume defined by the radiation oncologist [6]; (d) the applicators were covered with another layer of silicone and the remaining parts of the mask were also filled with this material in order to improve its durability. The mask is usually used from 8 to 10 days of treatment [7].

Such prepared mask should be left to dry completely, which may take even 24 hours. This period can be shortened by leaving the mask in an air-conditioned room. Then the mask should be carefully removed from the gypsum cast. The specialist should verify if the mask is correctly prepared.

Fiducial markers were placed on the borders of the lesion in order to facilitate the process of defining the planning target volume on CT images. We selected markers carefully, because we wanted to avoid any changes in mask positioning. There is another solution used to reach sites, i.e. cutting the inner layer of silicone and inserting the marker inside this cut. However, these cuts may need an additional thin layer of silicone to close them.

The mask was placed on the patient and fixed additionally with elastic dressings like Codofix or special Velcro tape for tracheal tubes or oxygen cannulas e.g. Posey foam track tie. Markers for CT/MRI examinations provided by Nucletron were placed in the needles. CT scout view allowed to define the range of CT scan and assess adhesion of the mask. CT images were sent to the treatment planning system TPS (Oncentra Brachy $4.0^{\circledR}$, Nucletron, an Elekta company, Elekta AB, Stockholm, Sweden) and the treatment plan was prepared. It was impossible to define any shape and size of protective plates in our TPS, therefore dose values for organs at risks were smaller in reality, and differences

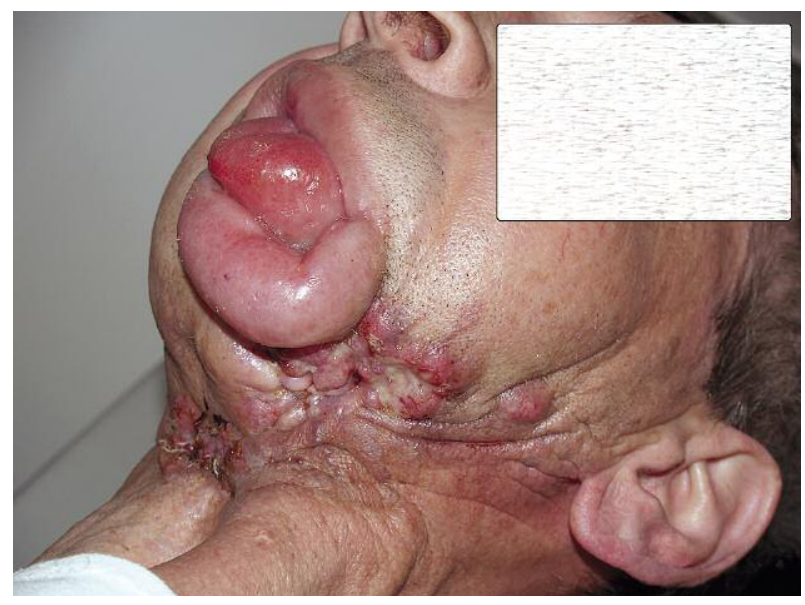

Fig. 4. Local recurrence in the skin of the neck, submandibular region (case 2 )

corresponded to plates' thickness and the material they were made of.

Protective plates should be repositioned on the mask before the first fraction of brachytherapy. They shouldn't affect the shape of the mask on the side of patient's skin. In order to have full control over the dose delivered to the planning target volume as well as organs at risk, especially when the eyeball is in a close proximity, in vivo dosimetry should be performed during the first fraction [8]. MOSFET detectors ${ }^{\circledR}$ (Best Medical Canada Ltd., Ottawa, Canada) may be used for this purpose. Micro-MOSFET detectors are very useful for small (approximately $1 \mathrm{~cm}^{2}$ ) and difficult to reach surfaces.

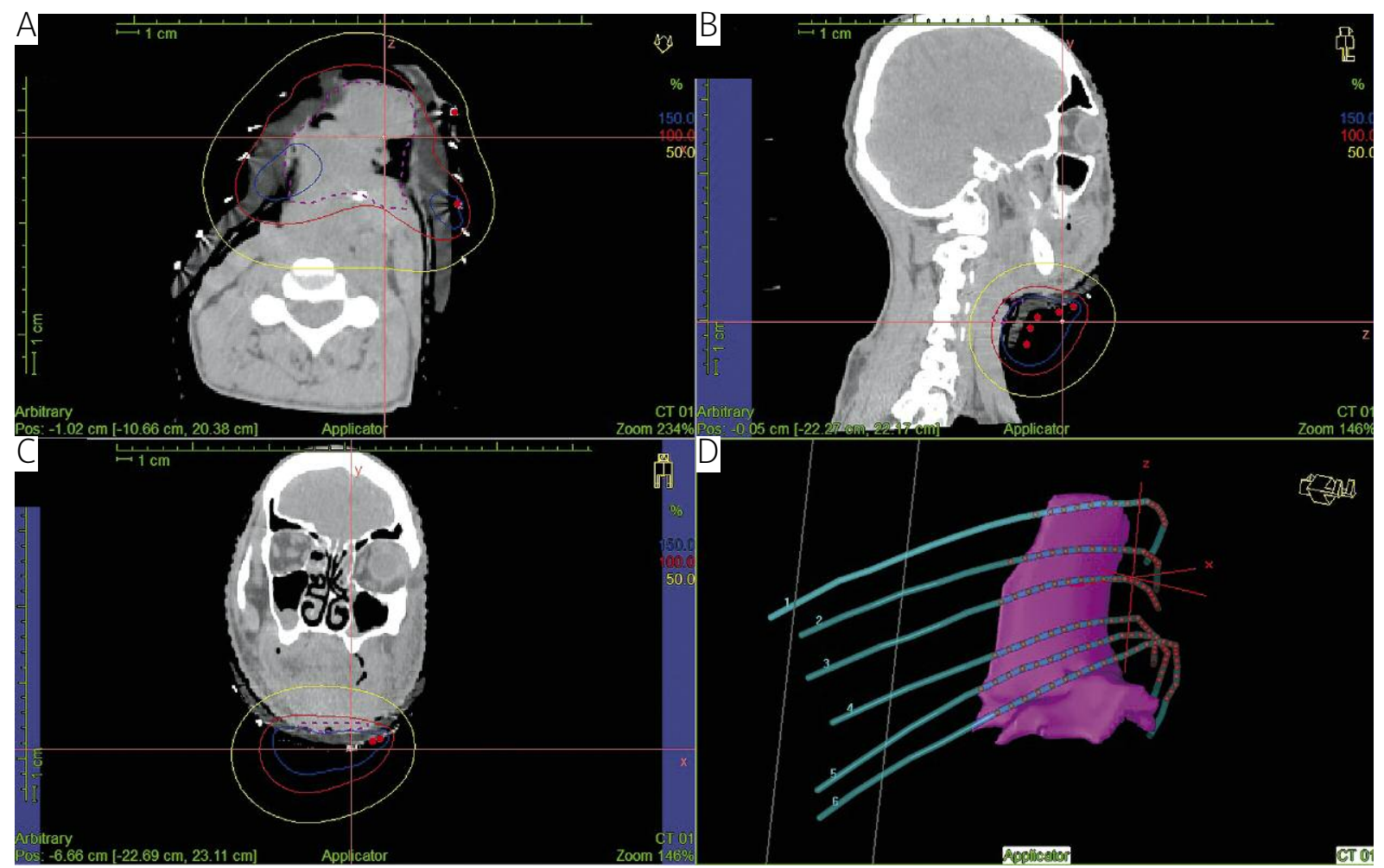

Fig. 5. CT images and reconstructions in different planes $(A, B, C)$ and a $3 D$ reconstruction (D) (case 2) 


\section{Description of cases}

\section{Case 1}

Patient with skin cancer (basal cell carcinoma), initially treated with EBRT (total dose of $70 \mathrm{~Gy}$ ) for nose location, then followed by stated recurrence on the nose and skin of the neck. Brachytherapy HDR was performed two years after primary treatment. Patient received 50 Gy in 10 fractions once a day.

\section{Case 2}

Primary cancer of the flour of the mouth (squamous cell carcinoma) radically treated with EBRT (total dose of $65 \mathrm{~Gy}$ ) combined with chemotherapy based on cisplatinum regiment. Local recurrence was found after three years on the skin of the neck on submandibular region. Patient received $30 \mathrm{~Gy}$ in 6 fractions once a day.

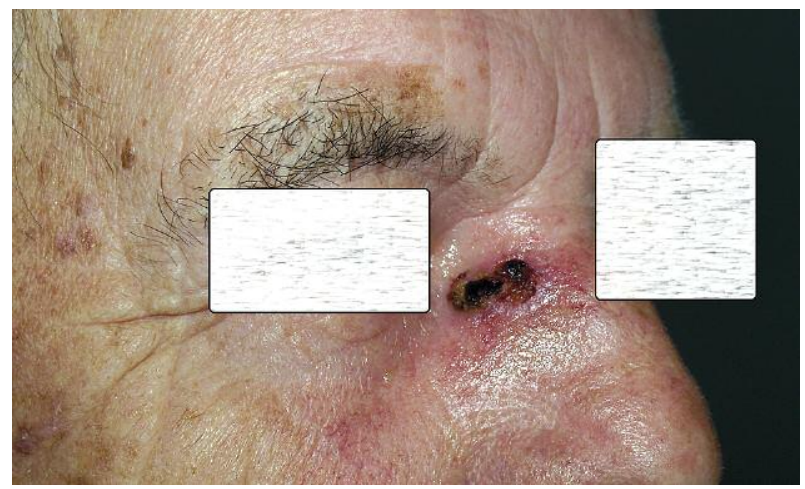

Fig. 6. A patient with locally advanced squamous cell carcinoma of the nose (case 3)

\section{Case 3}

Primary tumour on the skin (basal cell carcinoma), disqualified from the surgery, because of the general condition. Patient received 30 Gy in 6 fractions once a day.

Examples of individual applicators prepared for HDR brachytherapy are presented on following figures. View of lesions and treatment plan of a patient with multifocal head and neck cancer are presented in Figs. 1A-B and 2. In Fig. 3 CT images and reconstructions in different planes (A, B, C), and a $3 \mathrm{D}$ reconstruction (D) are presented. In Fig. 4 an applicator for case $\mathrm{nr} 2$ patient is presented; Fig. 5 shows treatment plan for this patient. Third case is a patient with locally advanced cancer in the skin of the nose (Fig. 6), corresponding treatment plan is presented in Fig. 7.

\section{Follow-up}

Patients described as cases $\mathrm{nr} 1$ and 3 have complete remission in 18 months after brachytherapy. In case nr 2 partial remission during 12 months of follow up was observed. After 12 months, rapid progression in glossary region was found. Patient was send to palliative chemotherapy.

\section{Discussion}

For the different types of the head and neck tumours EBRT \pm surgery is typically a treatment of choice. In some cases, especially in recurrent diseases or in location near eye bulb, EBRT or surgery is very risky, not acceptable for older patients. In this cases, brachytherapy seems to be more appropriate and effective method of treatment. For most of the patients, curative brachytherapy with individual mould

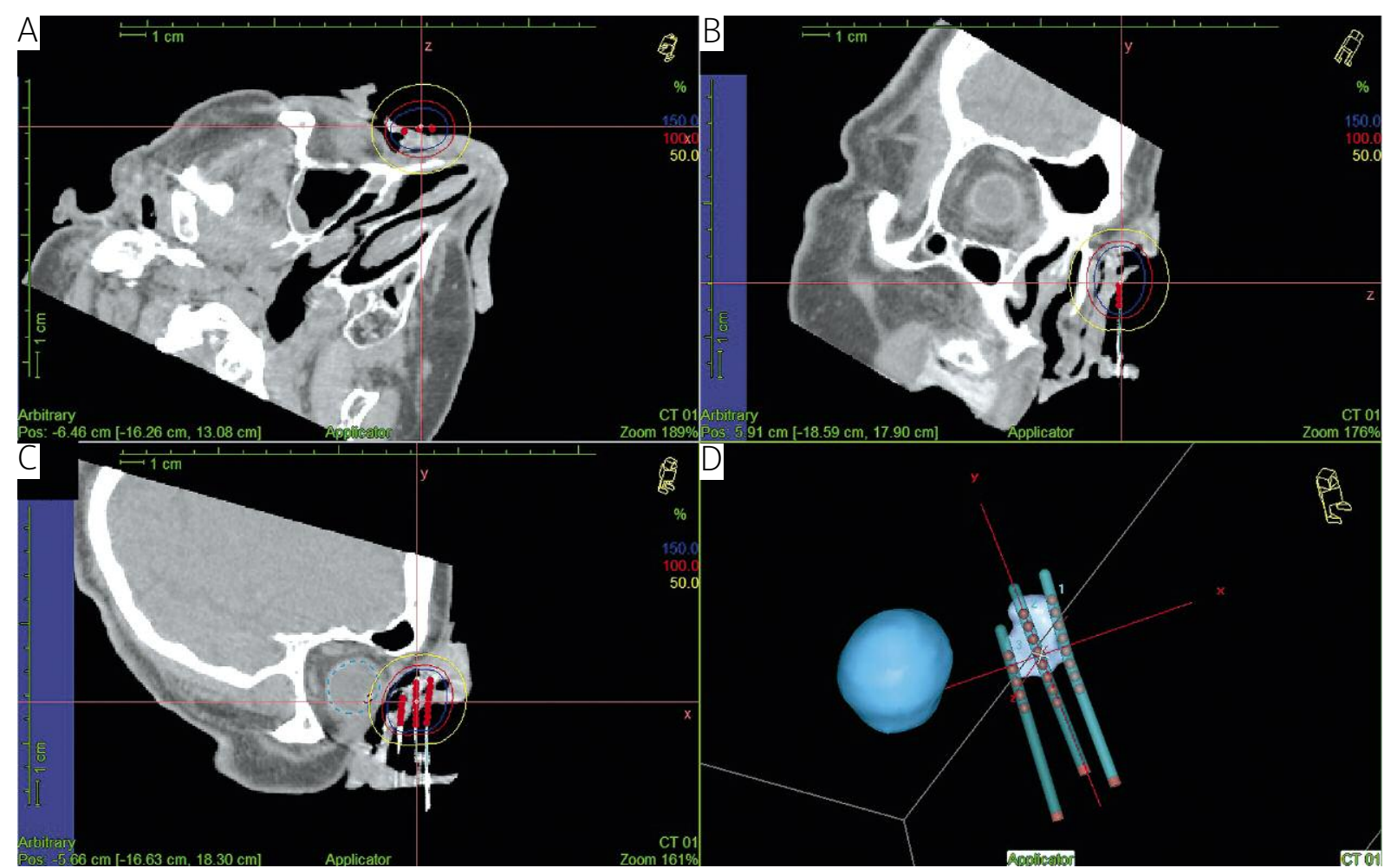

Fig. 7. CT images and reconstructions in different planes (A, B, C) and a 3D reconstruction (D) (case 3) 
applicator is much better tolerated than EBRT. Based on the published papers $[1,4,7]$, "focused" brachytherapy gives the patients better results with good tolerance and respectively less early or late sites effects. Individual brachytherapy performed in the localization of penile cancer [4] seems to be only one possibility to protect organ without severe sites effects with full protect functionality. In locations like head and neck region, brachytherapy offers a chance for complete remission of the disease without deep and prolonged sites effects like after externally irradiation [3,7].

\section{Conclusions}

Preparing individual applicator described in this article is technically very easy and focused on the individual clinical situation. In cases with local recurrences, especially in the superficial region, brachytherapy techniques seems to be the most save and easy treatment to protect organs located deeper than $2 \mathrm{~cm}$ below the surface, and from that reasons protecting critical healthy organs as well. Choosing HDR brachytherapy as a method of treatment, especially of the treatment regime, we must remember that superficial sites effects might be very severe. In our experience, sides effects prolongs 2-3 weeks after treatment, and with proper treatment (antibiotics applied locally) have no influences for longer follow up.

\section{References}

1. Guix B, Finestres F, Tello J et al. Treatment of skin carcinomas of the face by high-dose-rate brachytherapy and custom-made surface moulds. Int J Radiat Oncol Biol Phys 2000; 47: 95-102.

2. Applicator Guide, Applicators and Accessories for HDR \& PDR Brachytherapy, Nucletron BV, 2011.

3. Mazeron J, Ardiet J, Haie-Méder C. GEC-ESTRO recommendations for brachytherapy for head and neck squamous cell carcinomas. Radiother Oncol 2009; 91: 150-156.

4. Matys R, Kubicka-Mendak I, Łyczek J. Penile cancer brachytherapy HDR mould technique used at the Holycross Cancer Center. J Contemp Brachytherapy 2011; 3: 224-229.

5. Baltas D, Sakelliou L, Zamboglou N. The Physics of Modern Brachytherapy for Oncology. Taylor \& Francis, Boca Raton 2007.

6. Musmacher J, Ghaly M, Satchwill K. High dose rate brachytherapy with surface applicators: Treatment for non-melanomatous skin cancer. J Clin Oncol 2006; 24: S15543.

7. Owczarek G, Białas B, Smolska B et al. Our experience of mould brachytherapy technique for basal cell carcinoma of the face. J Contemp Brachytherapy 2009; 1: 188-189.

8. Sabbas AM, Kulidzhanov FG, Presser J et al. HDR brachytherapy with surface applicators: technical considerations and dosimetry. Technol Cancer Res Treat 2004; 3: 259-267. 